\title{
Cross-Canada survey of resistance of 2747 aerobic blood culture isolates to piperacillin/tazobactam and other antibiotics
}

Kevin R Forward MD FRCPC, Patricia A Franks PhD, Donald E Low MD FRCPC, Robert Rennie PhD, Andrew E Simor MD FRCPC and the Canadian Piperacillin/Tazobactam Study Group

KR Forward, PA Franks, DE Low, R Rennie, AE Simor, and the Canadian Piperacillin/Tazobactam Study Group. Cross-Canada survey of resistance of 2747 aerobic blood culture isolates to piperacillin/tazobactam and other antibiotics. Can J Infect Dis 1998;9(1):33-44.

OBJECTIVE: To compare the activity of piperacillin/tazobactam with that of other broad parenteral antibiotics against aerobic and facultative anaerobic blood culture isolates in a Canada-wide survey.

DESIGN: Fifty-eight laboratories in nine provinces each contributed up to 50 consecutive clinically significant aerobic and facultative anaerobic isolates for susceptibility testing.

SETTING: Participating hospitals included both tertiary care and community hospitals.

MATERIALS AND METHODS: Testing was performed in five regional centres by using the same microbroth dilution method, and results were interpreted according to National Commitee for Clinical Laboratory Standards M7-A3 and M100-S5 guidelines.

RESULTS: Piperacillin/tazobactam and imipenem were both active against more than $99 \%$ of the 1616 strains of Enterobacteriaceae species tested. The minimum inhibitory concentration of $90 \%$ of isolates (MIC 90 ) of all Enterobacteriaceae species was $2 \mathrm{mg} / \mathrm{L}$ for piperacillin/tazobactam compared with $64 \mathrm{mg} / \mathrm{L}$ for piperacillin alone. Seventeen per cent of strains of Enterobacteriaceae species were susceptible to piperacillin/tazobactam but resistant to piperacillin. Piperacillin/tazobactam was highly active against Pseudomonas aeruginosa, inhibiting $99.1 \%$ of strains. MIC 90 was $8 \mathrm{mg} / \mathrm{L}$. Nine per cent of $P$ aeruginosa strains were not susceptible to imipenem. Most of these strains had a MIC of $8 \mathrm{mg} / \mathrm{L}$, which falls in the intermediate category. Ninety-seven per cent of $P$ aeruginosa were susceptible to ciprofloxacin and $97.3 \%$ to tobramycin. Ninety-six per cent of strains of Actinobacter species were susceptible to piperacillin/tazobactam, whereas only $76 \%$ of strains were susceptible to piperacillin alone. Overall, piperacillin/tazobactam was the most active agent tested; $98 \%$ of all strains were susceptible, followed closely by imipenem, to which $97.8 \%$ of strains were susceptible. CONCLUSIONS: Aerobic blood culture isolates from Canadian centres continue to be highly susceptible to a variety of antibiotics. The broad spectrum of activity of piperacillin/tazobactam suggests that this combination should be considered for empirical treatment of sepsis while awaiting results of cultures and susceptibility testing.

Key Words: Antibiotic resistance, Piperacillin, Tazobactam, Susceptibility survery

Division of Microbiology, Department of Pathology and Laboratory Medicine, Queen Elizabeth II Health Sciences Centre, Halifax, Nova Scotia; Department of Microbiology and Infectious Diseases, Hôpital Maisoneuve-Rosemont, Montréal, Québec; Microbiology and Public Health, University of Alberta Hospital, Edmonton, Alberta; Wyeth-Ayerst Canada Inc; Department of Microbiology, Mount Sinai \& Princess Margaret Hospitals; Department of Microbiology, Sunnybrook Health Science Centre, Toronto, Ontario

Correspondence and reprints: Dr Kevin Forward, Service Chief, Division of Microbiology, Queen Elizabeth II Health Sciences Centre, 5788 University Avenue, Halifax, Nova Scotia B3H 1V8. Telephone 902-473-4109, fax 902-473-4432, e-mail Kevin.Forward@dal.ca

Accepted for publication January 28, 1997. Accepted April 1, 1997 


\section{Enquête pancanadienne sur la résistance de $\mathbf{2} 747$ isolats d'hémocultures aérobies à l'endroit du pipéracilline/tazobactam et d'autres antibiotiques}

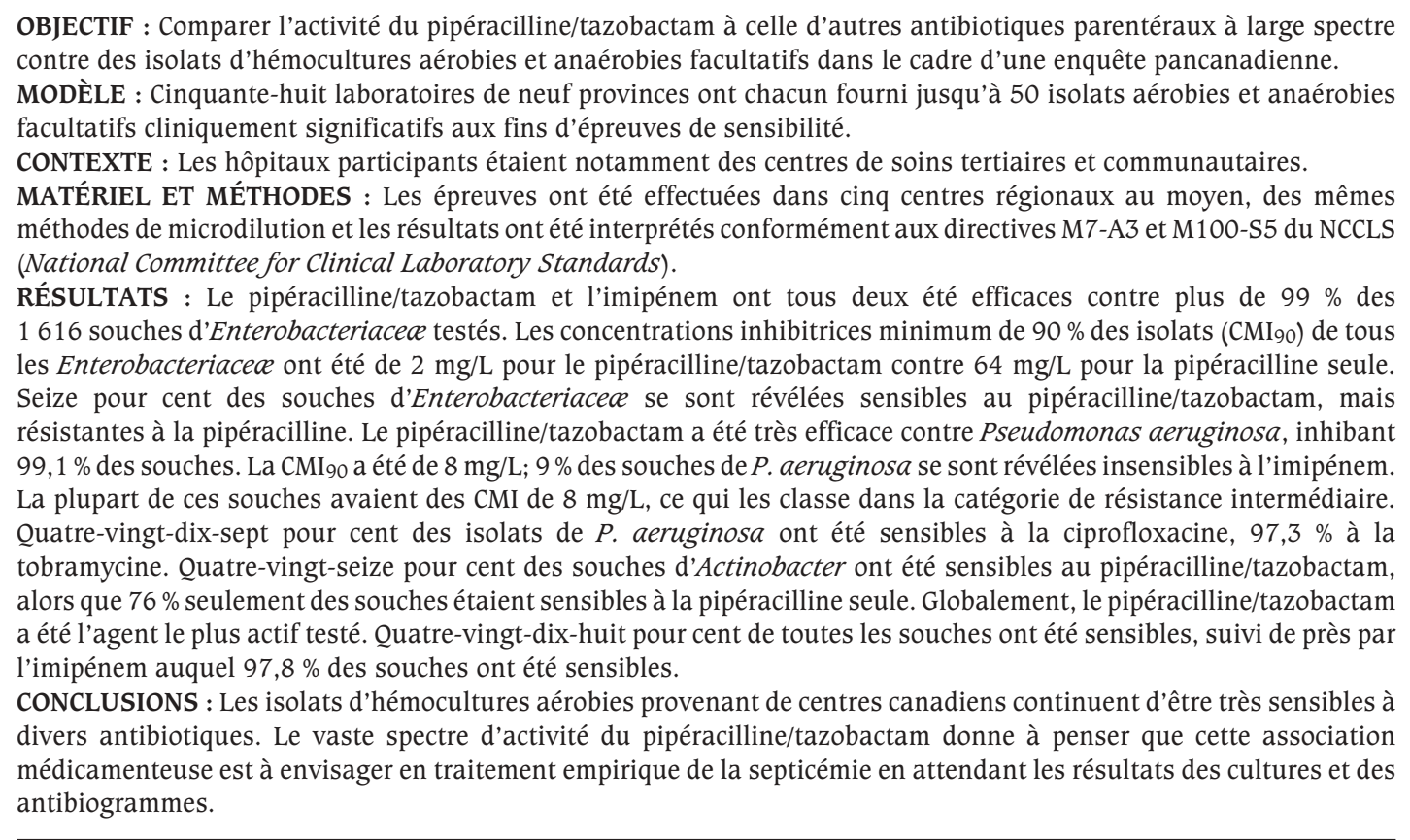

$\mathrm{P}$ iperacillin/tazobactam is the most recent of a number of beta-lactam/beta-lactamase inhibitor combinations to become available to Canadian physicians. The combination offers a number of advantages over previously marketed compounds. Piperacillin has a broader spectrum of activity than either the amino or carboxy penicillins, and tazobactam is a potent inhibitor of both chromosomally mediated and plasmid-mediated beta-lactamases $(1,2,3)$.

Numerous clinical trials have demonstrated the efficacy of piperacillin/tazobactam in the treatment of a variety of infections, including intra-abdominal infections, skin and skin structure infections, pelvic infections in women, pneumonia and fever in neutropenic patients undergoing chemotherapy for both solid and hematological malignancies (4-13). Noncomparative trials have also evaluated the efficacy of piperacillin/tazobactam with or without an aminoglycoside for the treatment of bacteremia $(14,15)$.

We conducted a prospective study of susceptibilities of blood culture isolates collected in 1995 from 58 sites across Canada to determine the prevalence of resistance to commonly used broad spectrum antibiotics, including piperacillin/tazobactam. Such data may help to establish the degree of confidence with which single agents may be used to treat serious bacteremic infections.

\section{MATERIALS AND METHODS}

Strains: Fifty-eight teaching and community hospitals from nine Canadian provinces participated in the evaluation. In most cases, 50 consecutive aerobic cultures were collected, beginning in February 1995. A few smaller centres fell short of this goal. Methicillin-resistant Staphylococcus species and anaerobes were not included. Potential contaminants, such as coagulase-negative Staphylococcus species and viridans streptococci, were submitted only if recovered on three different occasions from the same patient within one week. Only one strain from each species was submitted per patient.

Methods: Broth microdilution susceptibility studies were performed with 96-well microtitre plates containing serial twofold dilutions of study antibiotics. Panels were prepared by Sensititre (AccuMed International Inc, Ohio) and distributed to each of five regional testing centres. Organisms were speciated in their originating laboratory by using standard methods. Nonfastidious organisms were tested in accordance with National Committee for Clinical Laboratory Standards (NCCLS) guidelines (NCCLS M7-A3 and M100-S5) (16,17). Inocula were prepared by directly inoculating four to five colonies of an overnight culture of the organism incubated at 35 to $37^{\circ} \mathrm{C}$ into demineralized water to produce a suspension corresponding to a 0.5 McFarland standard. Ten microlitres of inoculum was suspended in $10 \mathrm{~mL}$ of cation-supplemented Mueller Hinton broth. Fifty microlitres of the resulting suspension was transferred to each well of the microtitre plates, which were then incubated aerobically at 34 to $36^{\circ} \mathrm{C}$ for $18 \mathrm{~h}$.

For susceptibility testing of Streptococcus pneumoniae, the inoculum was prepared in Mueller Hinton broth from overnight growth on $5 \%$ sheep blood agar. After mixing, $100 \mu \mathrm{L}$ of inoculum was added to $10 \mathrm{~mL}$ of cation-supplemented Mueller Hinton broth with added 2\% to 5\% lysed horse blood (PML Microbiological, Oregon). One hundred microlitres was inoculated to each well of the microtitre panel with an autoinoculator. Plates were incubated aerobically at $35^{\circ} \mathrm{C}$ for 20 to $24 \mathrm{~h}$.

Susceptibility testing for streptococci other than $S$ pneumoniae was performed as for $S$ pneumoniae except that only 10 $\mu \mathrm{L}$ was added to $10 \mathrm{~mL}$ of Mueller Hinton broth and $50 \mu \mathrm{L}$ was added to each well.

For Haemophilus influenzae, the inoculum was prepared in 
TABLE 1

Overall proportion of strains susceptible to antibiotics tested against all isolates in rank order

\begin{tabular}{lc}
\hline & \% susceptible \\
\hline Piperacillin-tazobactam & 98.0 \\
Piperacillin & 87.5 \\
Imipenem & 97.8 \\
Ceftazidime & 91.8 \\
Ciprofloxacin & 92.8 \\
Ticarcillin-clavulanate & 90.8 \\
Ceftriaxone & 91.5 \\
\hline
\end{tabular}

Mueller Hinton broth from isolated colonies on chocolate agar. Fifty microlitres of inoculum was transferred to $10 \mathrm{~mL}$ of Haemophilus species test medium broth (HTM) (BBL, Becton Dickinson and Company, Maryland). One hundred microlitres of HTM was added to each well. Plates were incubated at $35^{\circ} \mathrm{C}$ in carbon dioxide for 20 to $24 \mathrm{~h}$.

All testing was performed using the same lot number of panels, Haemophilus species test medium and cationsupplemented Mueller Hinton broth. American type culture collection (ATCC) organisms were tested as appropriate, with each batch of organisms run against both Gram-positive and Gram-negative microtitre panels - Staphylococcus aureus ATCC 29213, Enterococcus faecalis ATCC 29212 Escherichia coli ATCC 25922, E coli ATCC 35218 and Pseudomonas aeruginosa ATCC 27853.

\section{RESULTS}

Of the 2747 blood culture isolates tested, piperacillin/tazobactam was most active, inhibiting $98 \%$ of strains, followed closely by imipenem, which inhibited $97.8 \%$ of strains (Table 1). Ceftazidime, ceftriaxone, ciprofloxacin and ticarcillinclavulanate each inhibited over $90 \%$ of strains. Overall, the proportion of strains susceptible to third-generation cephalosporins and ciprofloxacin was lower than this percentage, primarily because of their intrinsic inactivity against enterococci, which comprised 160 of 2747 isolates. Against the 1611 strains of Enterobacteriaceae species tested, piperacillin/tazobactam was second only to imipenem in terms of the percentage of strains susceptible; only $0.2 \%$ of strains were resistant (Table 2). The minimum inhibitory concentration of 90\% of isolates ( MIC $_{90}$ ) of Enterobacteriaceae species was 2 $\mathrm{mg} / \mathrm{L}$ compared with $64 \mathrm{mg} / \mathrm{L}$ for piperacillin alone. Seventeen per cent of Enterobacteriaceae species were susceptible to piperacillin/tazobactam but resistant to piperacillin. Enterobacteriaceae species remained highly susceptible to all agents tested, with the exception of piperacillin and cefoxitin, which inhibited $82 \%$ and $81 \%$ of strains, respectively. Figure $1 \mathrm{com}-$ pares piperacillin/tazobactam with piperacillin MICs against strains of Enterobacteriaceae species, E coli, Enterobacter cloacae and Acinetobacter species. The majority of strains had lower piperacillin MICs in the presence of tazobactam than with piperacillin alone.
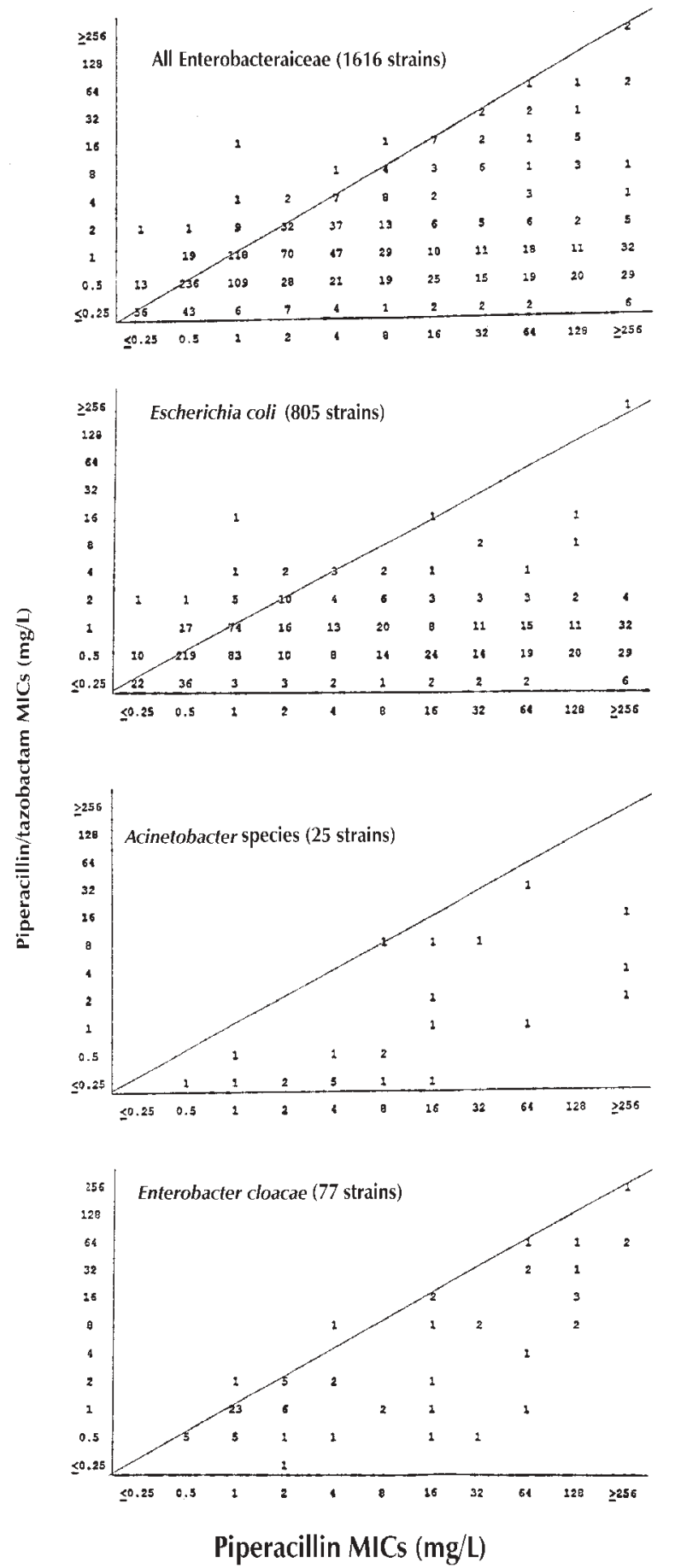

Figure 1) Comparison of the minimal inhibitory concentration (MIC) of piperacillin alone with that of piperacillin/tazobactam (8:1 ratio)

Piperacillin/tazobactam was the most active agent against $P$ aeruginosa, inhibiting $99.1 \%$ of strains; $\mathrm{MIC}_{90}$ was $8 \mathrm{mg} / \mathrm{L}$. Ninety-one per cent of strains were susceptible to imipenem and $8 \%$ had a MIC of $8 \mathrm{mg} / \mathrm{L}$, which fell into the intermediate category. Of the 111 strains of $P$ aeruginosa tested, $97.3 \%$ of 
TABLE 2

Comparative activity of piperacillin/tazobactam and other antibiotics against 2747 aerobic blood culture isolates

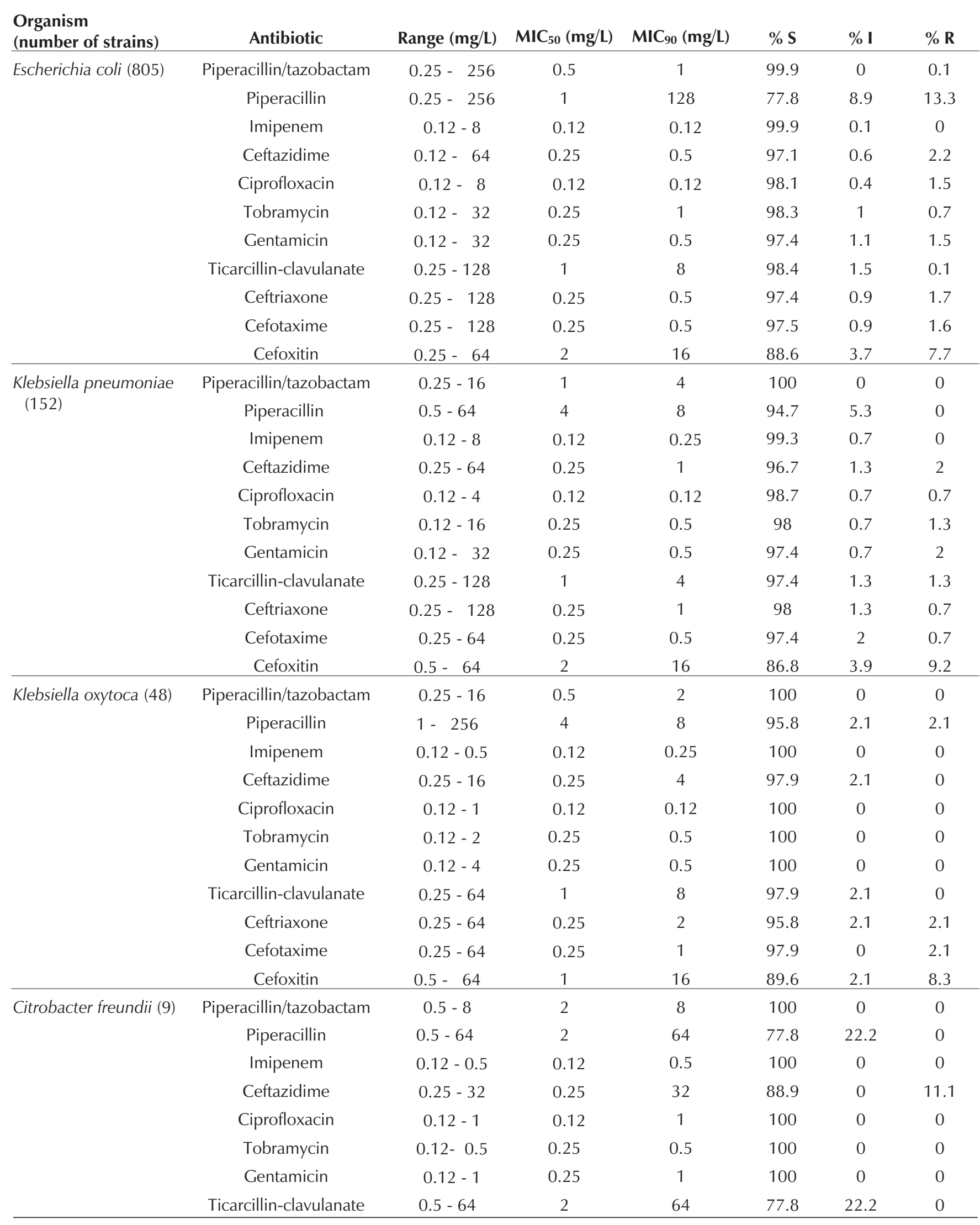


TABLE 2 continued

Comparative activity of piperacillin/tazobactam and other antibiotics against 2747 aerobic blood culture isolates

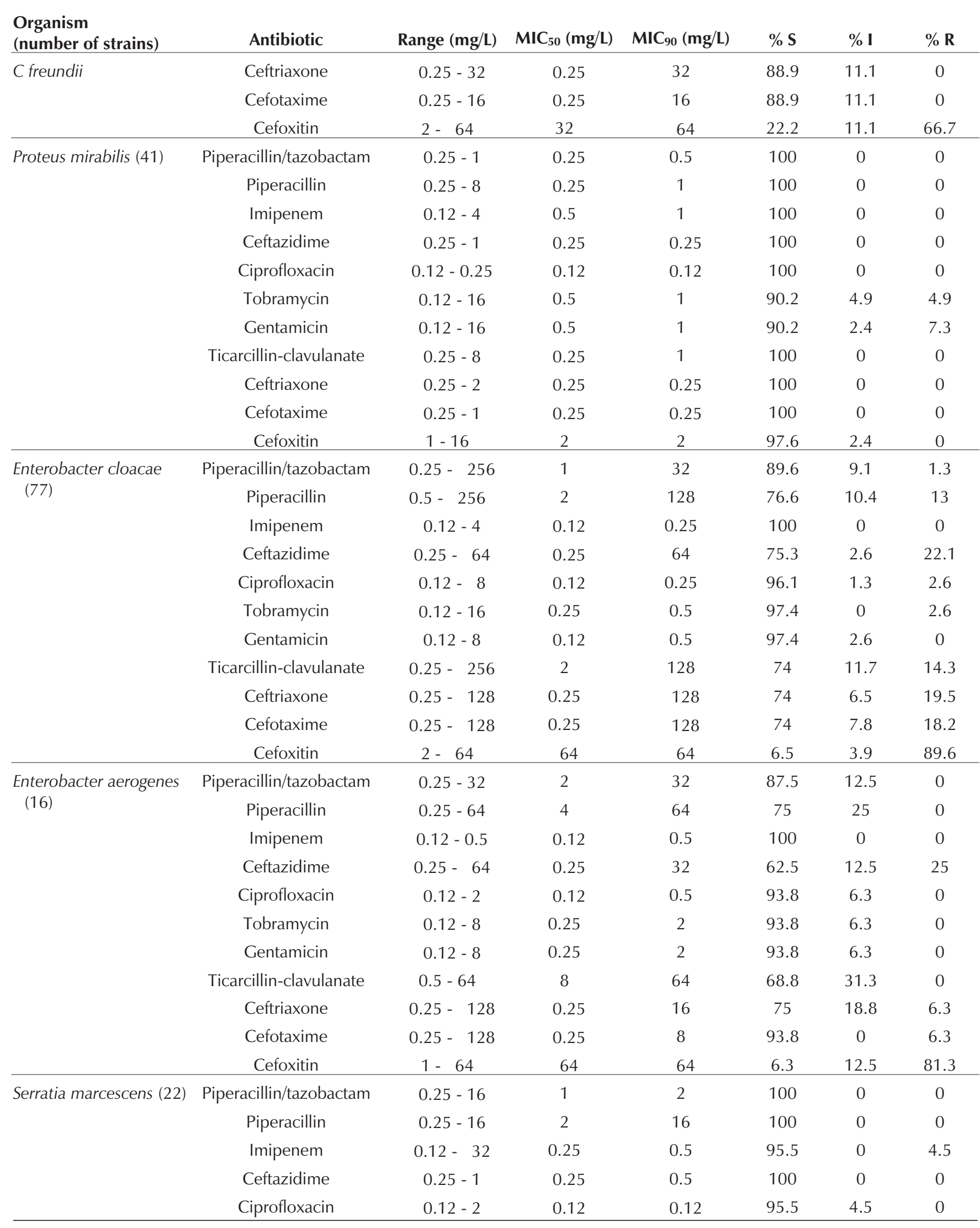


TABLE 2 continued

Comparative activity of piperacillin/tazobactam and other antibiotics against 2747 aerobic blood culture isolates

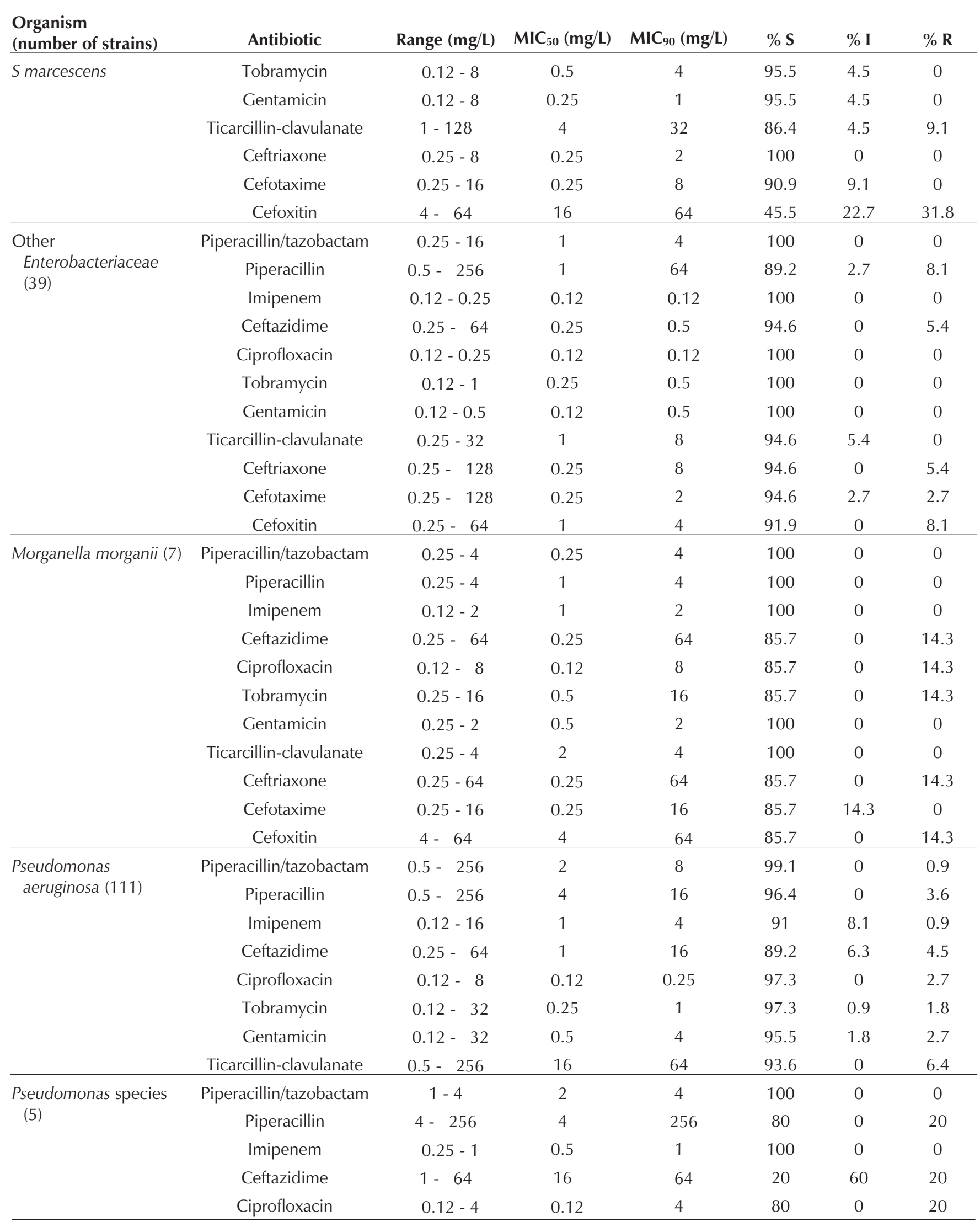


TABLE 2 continued

Comparative activity of piperacillin/tazobactam and other antibiotics against 2747 aerobic blood culture isolates

\begin{tabular}{|c|c|c|c|c|c|c|c|}
\hline $\begin{array}{l}\text { Organism } \\
\text { (number of strains) }\end{array}$ & Antibiotic & Range (mg/L) & $\mathrm{MIC}_{50}(\mathrm{mg} / \mathrm{L})$ & $\mathrm{MIC}_{90}(\mathrm{mg} / \mathrm{L})$ & $\% \mathrm{~S}$ & $\%$ I & $\% \mathbf{R}$ \\
\hline \multirow[t]{4}{*}{ Pseudomonas species } & Tobramycin & $0.25-1$ & 0.5 & 1 & 100 & 0 & 0 \\
\hline & Ticarcillin-clavulanate & $0.25-256$ & 64 & 256 & 20 & 40 & 40 \\
\hline & Ceftriaxone & $2-128$ & 8 & 128 & 60 & 0 & 40 \\
\hline & Cefoxitin & $8-64$ & 64 & 64 & 20 & 0 & 80 \\
\hline \multirow{8}{*}{$\begin{array}{l}\text { Acinetobacter species } \\
\quad(25)\end{array}$} & Piperacillin/tazobactam & $0.25-32$ & 0.5 & 8 & 96 & 4 & 0 \\
\hline & Piperacillin & $0.5-256$ & 8 & 256 & 76 & 12 & 12 \\
\hline & Imipenem & $0.12-32$ & 0.12 & 0.5 & 96 & 0 & 4 \\
\hline & Gentamicin & $0.12-32$ & 0.5 & 32 & 76 & 4 & 20 \\
\hline & Ticarcillin-clavulanate & $0.25-128$ & 4 & 64 & 84 & 12 & 4 \\
\hline & Ceftriaxone & $0.25-128$ & 8 & 64 & 68 & 20 & 12 \\
\hline & Cefotaxime & $0.25-64$ & 4 & 64 & 70.8 & 16.7 & 12.5 \\
\hline & Cefoxitin & $1-64$ & 64 & 64 & 28 & 4 & 68 \\
\hline \multirow{5}{*}{$\begin{array}{l}\text { Stenotrophomonas } \\
\text { maltophilia (13) }\end{array}$} & Piperacillin/tazobactam & $8-256$ & 8 & 256 & 61.5 & 0 & 38.5 \\
\hline & Piperacillin & $8-256$ & 32 & 256 & 38.5 & 23.1 & 38.5 \\
\hline & Imipenem & 32 & 32 & 32 & 0 & 0 & 100 \\
\hline & Cefotaxime & $2-128$ & 32 & 128 & 15.4 & 46.2 & 38.5 \\
\hline & Cefoxitin & 64 & 64 & 64 & 0 & 0 & 100 \\
\hline \multirow{9}{*}{$\begin{array}{l}\text { Haemophilus } \\
\text { influenzae (27) }\end{array}$} & Piperacillin/tazobactam & $0.25-0.25$ & 0.25 & 0.25 & 100 & 0 & 0 \\
\hline & Piperacillin & $0.25-64$ & 0.25 & 2 & 88.9 & 0 & 11.1 \\
\hline & Imipenem & $0.12-0.25$ & 0.12 & 0.25 & 100 & 0 & 0 \\
\hline & Ceftazidime & $0.25-0.25$ & 0.25 & 0.25 & 100 & 0 & 0 \\
\hline & Ciprofloxacin & $0.12-0.12$ & 0.12 & 0.12 & 100 & 0 & 0 \\
\hline & Ticarcillin-clavulanate & $0.25-0.5$ & 0.25 & 0.25 & 100 & 0 & 0 \\
\hline & Ceftriaxone & $0.25-0.25$ & 0.25 & 0.25 & 100 & 0 & 0 \\
\hline & Cefotaxime & $0.25-0.25$ & 0.25 & 0.25 & 100 & 0 & 0 \\
\hline & Cefoxitin & $0.25-2$ & 1 & 2 & 100 & 0 & 0 \\
\hline \multirow{4}{*}{$\begin{array}{l}\text { Other Gram-negative } \\
\text { rods ( } 7)\end{array}$} & Piperacillin/tazobactam & $0.25-16$ & 0.5 & 16 & 100 & 0 & 0 \\
\hline & Piperacillin & $0.25-64$ & 1 & 64 & 71.4 & 28.6 & 0 \\
\hline & Imipenem & $0.12-0.5$ & 0.12 & 0.5 & 100 & 0 & 0 \\
\hline & Ceftazidime & $0.25-16$ & 0.5 & 16 & 85.7 & 14.3 & 0 \\
\hline
\end{tabular}


TABLE 2 continued

Comparative activity of piperacillin/tazobactam and other antibiotics against 2747 aerobic blood culture isolates

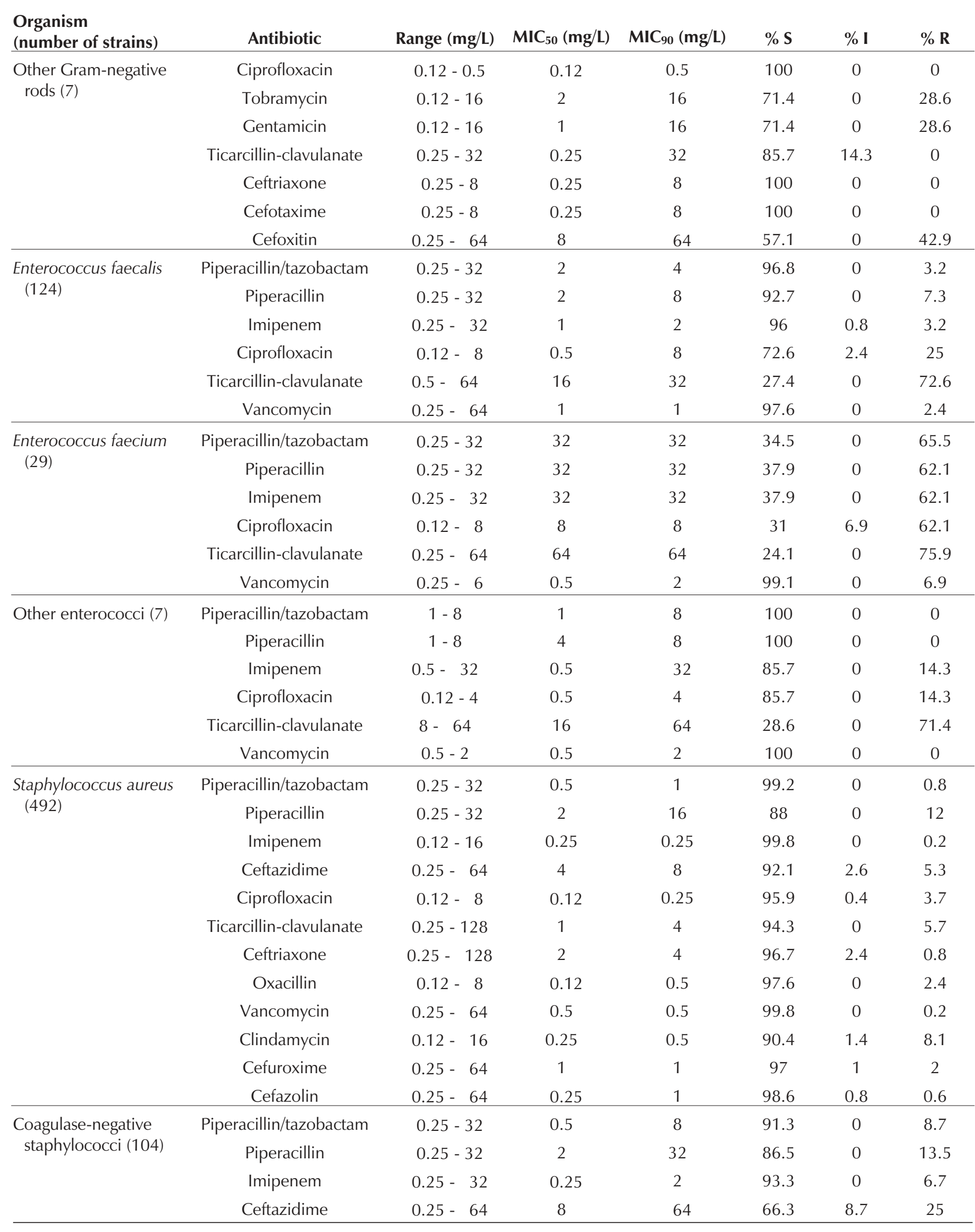


TABLE 2 continued

Comparative activity of piperacillin/tazobactam and other antibiotics against 2747 aerobic blood culture isolates

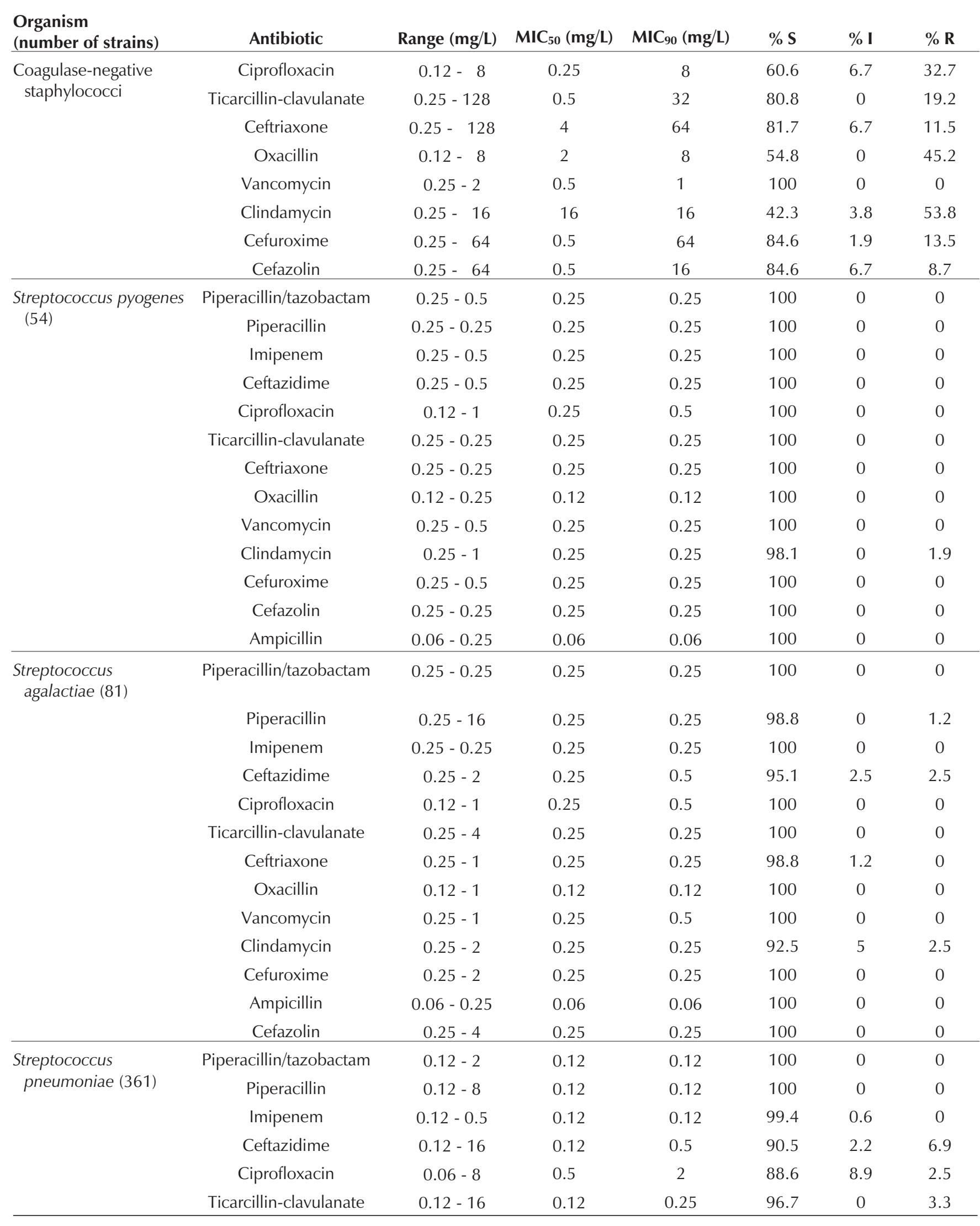


TABLE 2 continued

Comparative activity of piperacillin/tazobactam and other antibiotics against 2747 aerobic blood culture isolates

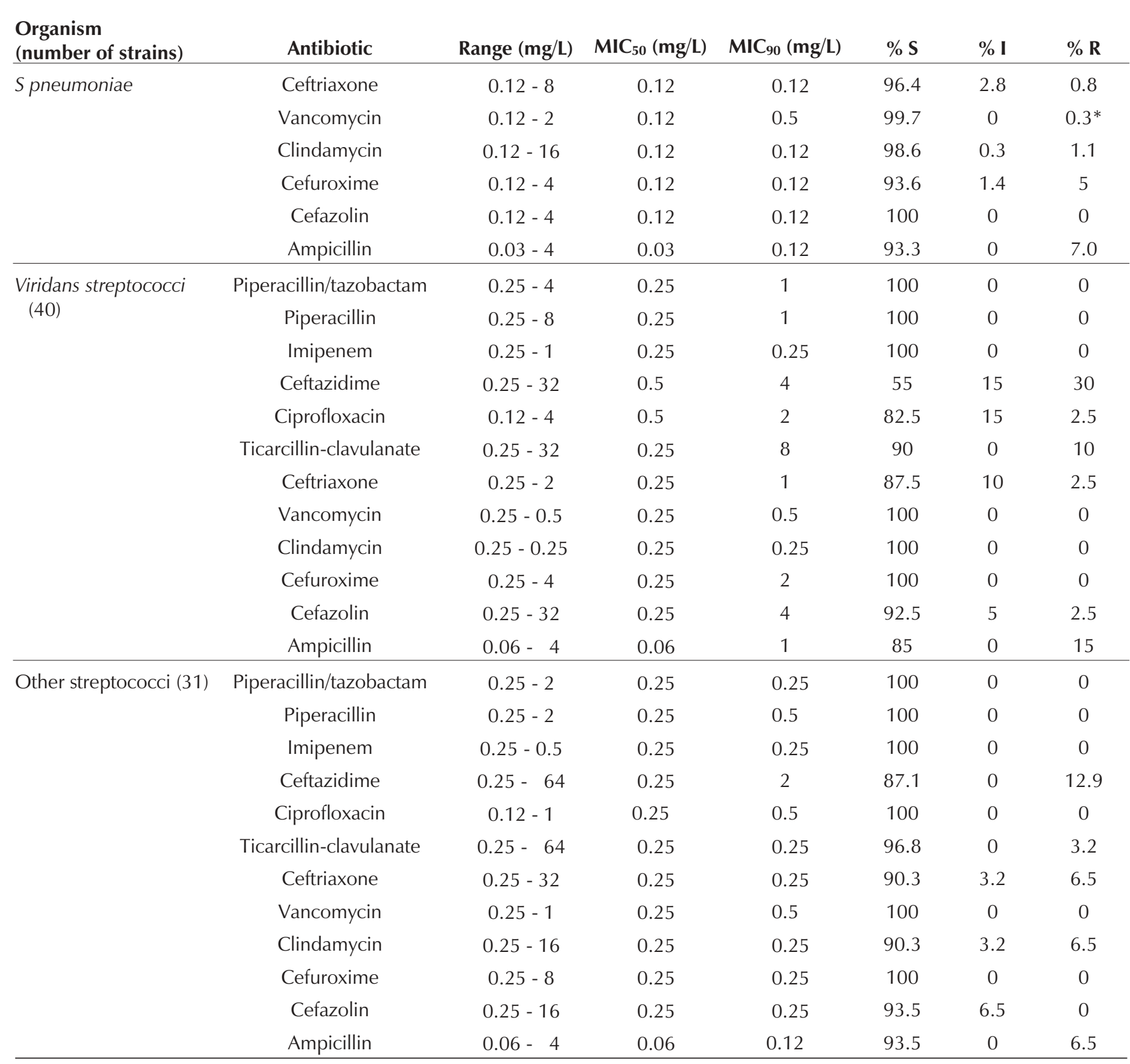

I Intermediate; MIC 50 Minimum inhibitory concentration required to inhibit 50\% of isolates; $\mathrm{MIC}_{90}$ Minimum inhibitory concentration required to inhibit $90 \%$ of isolates; R Resistant; S Susceptible

strains were susceptible to ciprofloxacin. Canadian strains remain highly susceptible to aminoglycosides, the $\mathrm{MIC}_{90}$ for tobramycin being only $1 \mathrm{mg} / \mathrm{L}$.

Twenty-five strains of Acinetobacter species were tested and $96 \%$ of these were susceptible to piperacillin/tazobactam compared with only $76 \%$ of strains susceptible to piperacillin. Only $76 \%$ of strains were susceptible to aminoglycosides, and only $68 \%$ were susceptible to ceftriaxone. Ceftazidime was somewhat more active than ceftriaxone ( $\mathrm{MIC}_{90} 8 \mathrm{mg} / \mathrm{L}$ versus $64 \mathrm{mg} / \mathrm{L}$ ) against Acinetobacter species.
All 361 strains of $S$ pneumoniae were susceptible to piperacillin/tazobactam, piperacillin and imipenem. The highest observed piperacillin/tazobactam MIC was $1 \mathrm{mg} / \mathrm{L}$. The highest imipenem MIC was $0.25 \mathrm{mg} / \mathrm{L}$. Only two ceftriaxone-resistant strains (MIC $2 \mathrm{mg} / \mathrm{L}$ or greater) were identified. The highest ceftriaxone MIC observed was $8 \mathrm{mg} / \mathrm{L}$. Of the $S$ pneumoniae strains tested, $11.3 \%$ had decreased susceptibility to ciprofloxacin (MIC $2 \mathrm{mg} / \mathrm{L}$ or greater).

Only three vancomycin-resistant $E$ faecalis strains were identified, and only two of 29 Enterococcus faecium strains 
were vancomycin resistant. While piperacillin/tazobactam was highly active against $E$ faecalis, only $34.5 \%$ of $E$ faecium strains were susceptible.

\section{DISCUSSION}

The antibiotic armamentarium offers a number of choices for the initial empirical treatment of severely ill patients with bacteremia. The choice of agents in this setting should take into account the observed susceptibility patterns of isolates most frequently encountered. In this study, we compared the activity of a number of broad spectrum antibiotics with Canadian strains collected from both tertiary centres, and community and regional hospitals. With the exception of anaerobes and methicillin-resistant staphylococci, the numbers and proportion of strains tested should be quite representative of the Canadian experience. We have previously made the observation that Canadian strains are often more susceptible to antibiotics than those in Europe and the United States, and, therefore, it is important to use Canadian susceptibility data when making therapeutic choices (18).

of the agents tested, piperacillin/tazobactam and imipenem had the broadest spectrums of activity. We would also expect these agents to be active against the anaerobes that were recovered during the same period of time but were not tested (19). Of the strains tested, only E faecium and Stenotrophomonas maltophilia were frequently resistant to these agents E faecium, by virtue of altered penicillin binding proteins and $S$ maltophilia because of its broad spectrum metalloenzyme and decreased permeability.

Piperacillin/tazobactam was much more active against $E$ coli than piperacillin ( $\mathrm{MIC}_{90} 1 \mathrm{mg} / \mathrm{L}$ versus $128 \mathrm{mg} / \mathrm{L}$ ). Twenty-two per cent more strains were susceptible to piperacillin/tazobactam than to piperacillin alone. This presumably reflects the prevalence of TEM1 beta-lactamases in $E$ coli and their susceptibility to tazobactam (1). For all Enterobacteriaceae species tested, the $\mathrm{MIC}_{90}$ was lower for piperacillin/tazobactam than for piperacillin. Possibly, this represents a small amount of intrinsic activity of tazobactam, as well as its activity against beta-lactamases from a number of classes. We also observed that $E$ cloacae strains usually had lower MICs to piperacillin/tazobactam than to piperacillin. This has been observed by others and is likely a reflection of the weak activity of tazobactam against class 1 beta-lactamases (20). When piperacillin/tazobactam was compared with ticarcillin-clavulanate, only $S$ maltophilia was more susceptible to ticarcillinclavulanate ( $\mathrm{MIC}_{90} 64 \mathrm{mg} / \mathrm{L}$ versus $256 \mathrm{mg} / \mathrm{L}$ ). This has been noted by others (21-24).

A number of agents showed reduced activity against Acinetobacter species. Only $76 \%$ of strains were susceptible to gentamicin and tobramycin, and only $71 \%$ were susceptible to cefotaxime. Imipenem remained highly active against Acinetobacter species, the $\mathrm{MIC}_{90}$ being $0.5 \mathrm{mg} / \mathrm{L}$. Ninety-six per cent of strains were susceptible to imipenem. Piperacillin/tazobactam was more active than piperacillin (MIC $908 \mathrm{mg} / \mathrm{L}$ versus 256 $\mathrm{mg} / \mathrm{L}$ ), and $20 \%$ more strains were more susceptible to piperacillin/tazobactam. These findings likely reflect both the intrinsic

\section{Members of the Canadian Piperacillin/Tazobactam Study Group}

British Columbia: B Robinson, Lions Gate Hospital, North Vancouver; J Roy, Royal Columbia Hospital, New Westminster

Alberta: DL Church, Alberta Children's Hospital, Calgary; $J$ Galbraith, Royal Alexandra Hospital, Edmonton, Alberta; P Kibsey, Misericordia/Gray Nuns Hospital, Edmonton; ET Larsen, Rockyview General Hospital, Calgary; R Rennie, University of Alberta Hospital, Edmonton, Alberta; $K$ Ramotar, Calgary General Hospital, Calgary

Manitoba: G Harding, St Boniface General Hospital, Winnipeg; DJ Hoban, Health Sciences Centre, Winnipeg

Saskatchewan: JM Blondeau, St Paul's Hospital, Saskatoon; G Richards, Pasqua Hospital, Regina; G Richards, Plains Health Center, Regina; PAG Tilley, Royal University Hospital, Saskatoon

Ontario: I Campbell, The Toronto Hospital, Toronto; D Clarke, Kitchener-Waterloo Hospital, Kitchener; WD Colby, University Hospital, London; Mr M Davies, McKellar General Hospital, Thunder Bay; F Diaz-Mitoma, Children's Hospital of Eastern Ontario, Ottawa; H Dick, The Wellesley Hospital, Toronto; U Hammerberg, St Joseph's Health Centre, London; J Haworth, Joseph Brant Memorial Hospital, Burlington; $Z$ Hussein, Victoria Hospital, London; P Jessamine, Ottawa Civic Hospital, Ottawa; S Krajden, St Joseph's Health Centre, Toronto; D Low, Mount Sinai Hospital, Toronto; I Luchsinger, Hamilton General Hospital, Hamilton; B Moderski, North York General Hospital, North York; Z Moine, Peel Memorial Hospital, Brampton; H Richardson, McMaster University Medical Centre, Hamilton; S Richardson, Hospital for Sick Children, Toronto; A Simor, Sunnybrook Health Sciences Centre, Toronto; JH Thornley, Henderson General Division, Hamilton; B Toye, Ottawa General Hospital, Ottawa; D Zoutman, Kingston General Hospital, Kingston

Québec: L Cote, Centre Hôpital de l'Université Laval, Ste-Foy; $L$ Delorme, Charles Lamoyne Hospital, Greenfield Park; $P$ Dolce, Centre Hospital Region of Rimouski, Rimouski; $M$ Gourdeau, Hôpital de I'Enfant Jesus, Québec; P Hivon, Hôpital St François de'Assise, Québec; M Ishak, Hôtel-Dieu de Saint-Jérome, Saint-Jérome; P-J Laflamme, CHRDL, Joliette; M Laverdiere, Maisonneuve-Rosemont Hospital, Montreal; D Lymand, Centre Hospitalier Pierre-Boucher, Longeuil; M Miller, Jewish General Hospital, Montreal; I Morissettel D Lauzon, Hôpital du Haut Richelieu, St-Jean-sur-Richelieu; G Murray, Hôpital du Saint-Sacrement; J-F Paradis, Hôpital de Chicoutimi, Chicoutimi; S Peloquin, Centre Hospitalier Saint-Michel, Montréal; G Pichette, Hôpital Sacre-Coeur, Montréal; M Poisson, Hôtel-Dieu de Montréal, Montréal; A Vibien, Reseau Sante Richelieu-Yamaska, Saint-Hyacinthe

New Brunswick: M Kuhn, The Moncton Hospital, Moncton; P Leighton, Dr Everett Chalmers Hospital, Fredericton

Prince Edward Island: LP Abbott, Queen Elizabeth Hospital, Charlottetown

Nova Scotia: KR Forward, Queen Elizabeth II Health Sciences Centre, Halifax 
activity of tazobactam against Acinetobacter species and the prevalence of beta-lactamases in Acinetobacter species $(1,25)$.

\section{CONCLUSIONS}

Blood culture isolates from this Canada-wide study remain highly susceptible to a variety of commonly used antibiotics representing several different classes. Piperacillin and tazobactam together inhibited more than $98 \%$ of most organism groups tested, including Enterobacteriaceae species, $P$ aeruginosa, $H$ influenzae and streptococci. Piperacillin/tazobactam has a spectrum of activity that provides coverage for bloodstream infections in the vast majority of cases. Other factors, including the result of treatment trials, pharmacokinetics and pharmacoeconomics, will ultimately be required to define the place of piperacillin/tazobactam in the empirical treatment of suspected bacteremia.

\section{REFERENCES}

1. Bush K, Jacoby GA, Medeiros AA. A functional classification scheme for -lactamases and its correlation with molecular structure. Antimicrob Agents Chemother 1995;39:1211-33.

2. Bryson HM, Brogden RN. Piperacillin/tazobactam: a review of its antibacterial activity, pharmacokinetic properties and therapeutic potential. Drugs 1994;47:506-35.

3. Sanders WE, Sanders CC. Piperacillin-tazobactam: a critical review of the evolving clinical literature. Clin Infect Dis 1996;22:107-23.

4. Shlaes DM, Baughman R, Boylen CT, et al. Piperacillin/ tazobactam compared with ticarcillin/clavulanate in community-acquired bacterial lower respiratory tract infection. J Antimicrob Chemother 1994;3:565-77.

5. Sweet RL, Roy S, Faro S, O'Brien WF, Sanfilippo JS, Seidlin M. Piperacillin and tazobactam versus clindamycin and gentamicin in the treatment of hospitalized women with pelvic infection. Obstet Gynecol 1993;83:280-5.

6. Tan JS, Wishnow RM, Talan DA, Duncanson FP, Norden CW. Treatment of hospitalized patients with complicated skin and skin structure infections: double-blind, randomized, multi-centre study of piperacillin/tazobactam versus ticarcillin/clavulanate. The Piperacillin/Tazobactam Skin and Skin Structure Study Group. Antimicrob Agents Chemother 1993;37:1580-6.

7. Moulton Y, Leroy O, Beuscart C. Efficacy, safety and tolerance of parenteral piperacillin/tazobactam in the treatment of patients with lower respiratory tract infections. J Antimicrob Chemother 1993;31(Suppl A):87-95.

8. Gorbach SL. Piperacillin/tazobactam in the treatment of polymicrobial infections. Intensive Care Med 1994;20:S27-34.

9. Nowe P. Piperacillin/tazobactam in complicated urinary tract infections. Intensive Care Med 1994;20(Suppl 3):S39-42.

10. Brismar D, Malmborg AS, Tunevall G, et al. Piperacillintazobactam versus imipenem-cilastatin in the treatment of intra-abdominal infections. Antimicrob Agents Chemother 1992;36:2766-74.
11. Niinikoski J, Havia T, Alhava E et al. Piperacillin/tazobactam versus imipenem/cilastatin in the treatment of intra-abdominal infection. Surg Gynecol Obstet 1993;176:255-61.

12. Cometta A, Zinner S, de Bock R, et al. Piperacillin-tazobactam plus amikacin versus ceftazidime plus amikacin as empiric therapy for fever in granulocytopenic patients with cancer. The International Antimicrobial Therapy Cooperative Group of the European Organization for Research and Treatment of Cancer. Antimicrob Agents Chemother 1995;39:445-52.

13. Micozzi A, Nucci M, Venditti M, et al. Piperacillin/tazobactam/ amikacin versus piperacillin/amikacin/tiecoplanin in the empirical treatment of neutropenic patients. Eur J Clin Microbiol Infect Dis 1993;12:1-8.

14. Wise R. The efficacy and safety of piperacillin/tazobactam in the therapy of bacteremia. J Antimicrob Chemother 1993;31 (Suppl A):97-104.

15. Charbonneau P. Review of piperacillin/tazobactam in the treatment of bacteremic infections and summary of clinical efficacy. Intensive Care Med 1994;20:S43-8.

16. National Committee for Clinical Laboratory Standards (NCCLS) methods for dilution antimicrobial susceptility tests for bacteria that grow aerobically: approved standard M7-A3. Villanova, 1993.

17. National Committee for Clinical Laboratory Standards (NCCLS) Performance standards for antimicrobial susceptibility testing: 5th Informational Supplement, M100-S5. Villanova, 1994.

18. Forward KR, Low DE, Laverdiere M, et al. Study of the comparative activity of piperacillin/tazobactam with currently available antibiotics against 8206 aerobic isolates. Can J Infect Dis 1997;8:147-53.

19. Appelbaum PC, Spangler SK, Jacobs MR. Susceptibility of 539 gram-positive and gram-negative anaerobes to new agents, including RP59500, biapenem, trospectomycin and piperacillin/tazobactam. J Antimicrob Chemother 1993;32:223-31.

20. Akova M, Yang Y, Livermore DM. Interactions of tazobactam and clavulanate with inducibly and constitutively expressed Class I beta-lactamases. J Antimicrob Chemother 1990;25:199-208.

21. Knapp CC, Sierra-Madero J, Washington JA. Activity of ticarcillin/clavulanate and piperacillin/tazobactam (YTR 830; CL-298, 741) against clinical isolates and against mutants derepressed for class I beta-lactamase. Diagn Microbiol Infect Dis 1989;12:511-5.

22. Fass RJ, Prior RB. Comparative in vitro activities of piperacillintazobactam and ticarcillin-clavulanate. Antimicrob Agents Chemother 1989;33:1268-74.

23. Eliopoulos GM, Klimm K, Ferraro MJ, Jacoby GA, Moellering RC Jr. Comparative in vitro activity of piperacillin combined with the beta-lactamase inhibitor tazobactam (YTR 830). Diagn Microbiol Infect Dis 1989;12:481-8.

24. Garcia-Rodriguez JA, Garcia Sanchez JE, Garcia Garcia MI, Garcia Sanchez E, Munoz Bellido JL. Antibiotic susceptibility profile of Xanthomonas maltophilia in vitro activity of

-lactam/ -lactamase inhibitor combinations. Diagn Microbiol Infect Dis 1991;14:239-43.

25. Suh B, Shapiro T, Jones R, Satishchandran V, Truant AL. In vitro activity of -lactamase inhibitors against clinical isolates of Acinetobacter species. Diagn Microbiol Infect Dis 1995;21:111-4. 


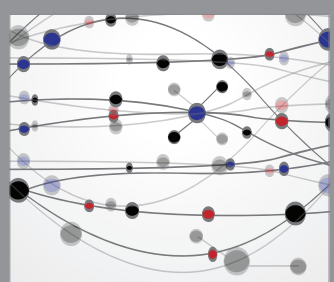

The Scientific World Journal
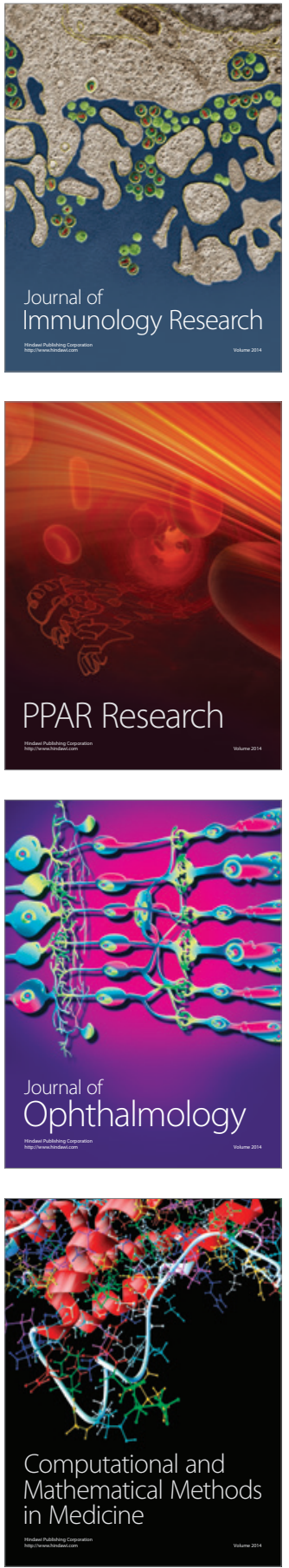

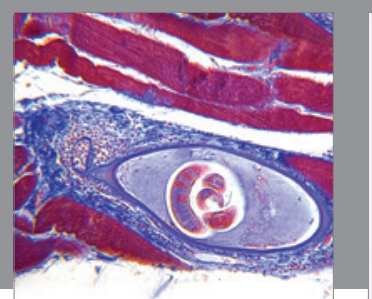

Gastroenterology Research and Practice

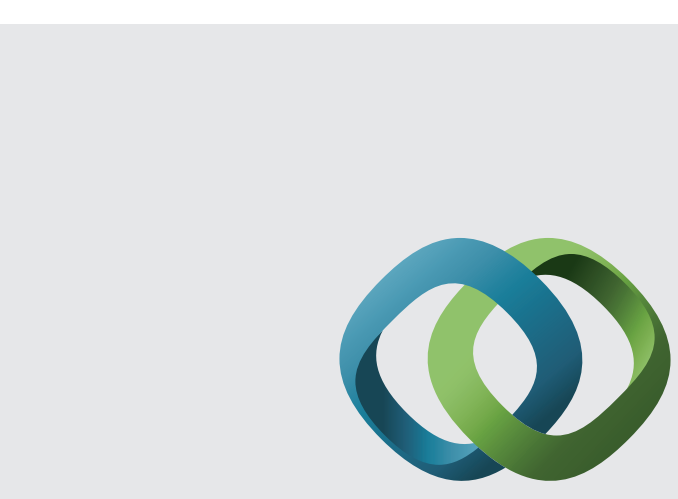

\section{Hindawi}

Submit your manuscripts at

http://www.hindawi.com
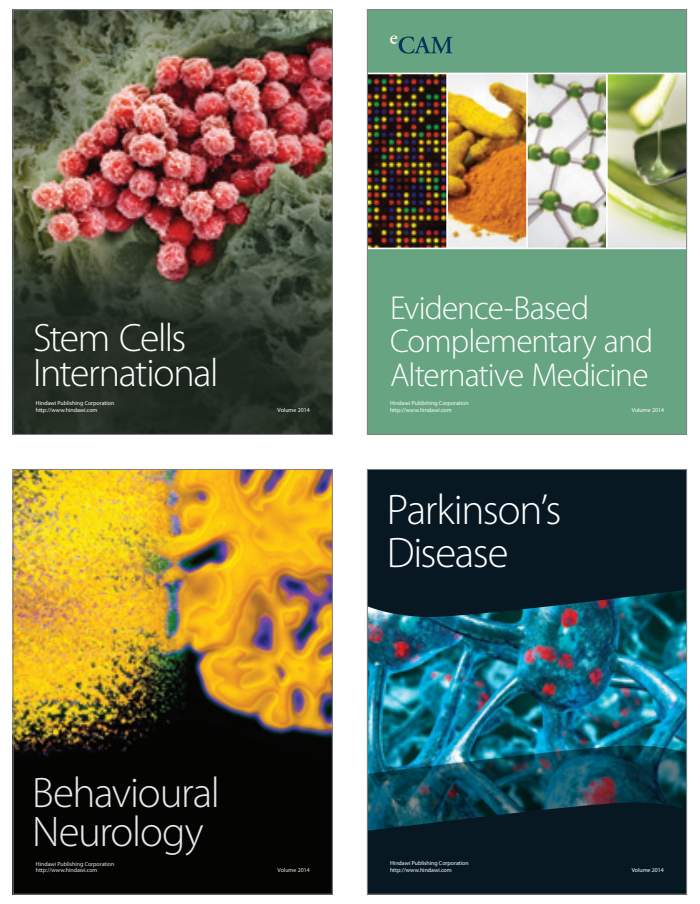
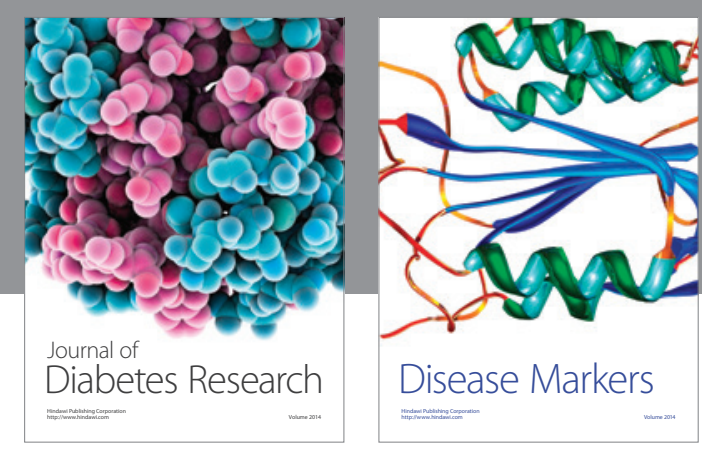

Disease Markers
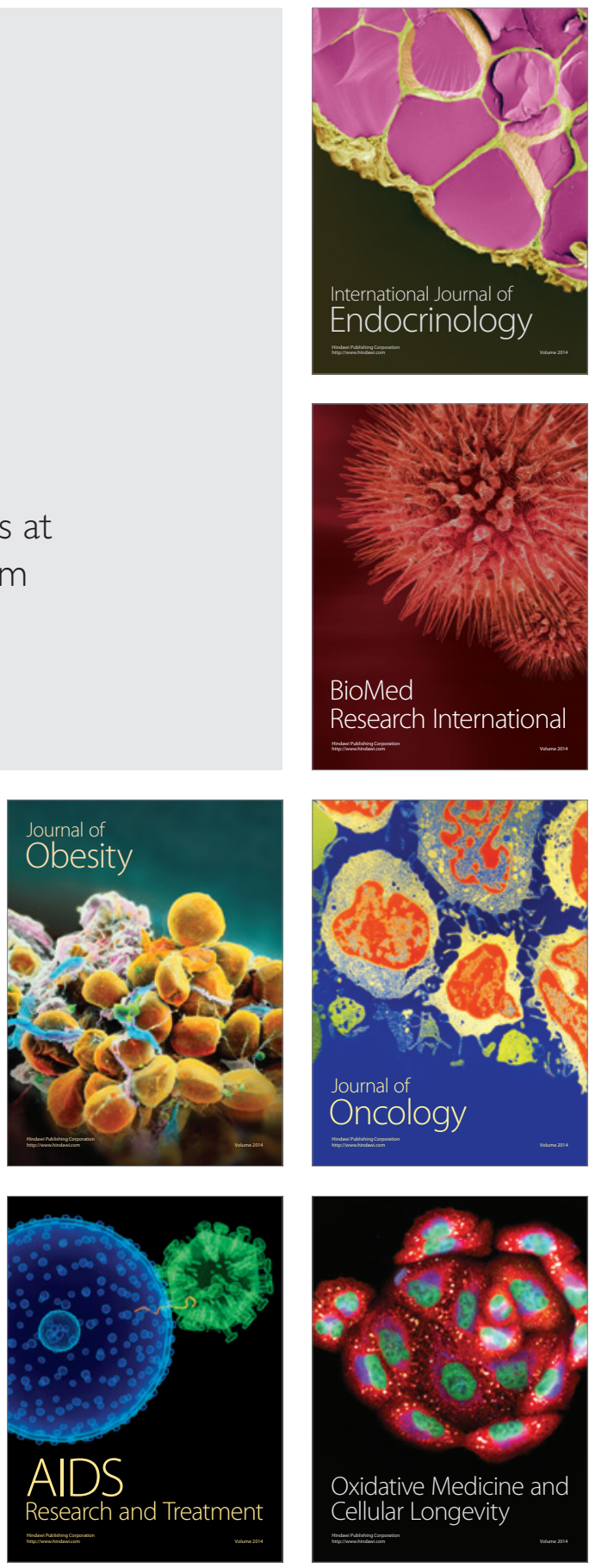